Koloskopieintervalle überdenken

\title{
Wie oft zum Check nach Polypektomie?
}

Innerhalb von fünf Jahren nach einer Polypektomie ist das Risiko für ein erneutes kolorektales Karzinom bei allen Patienten gering, so eine deutsche Fall-Kontroll-Studie.

Fast bei jeder dritten Erstkoloskopie werden Adenome entdeckt und entfernt. In welchen Intervallen die Patienten dann allerdings nachuntersucht werden sollen - darüber liegen bislang noch wenige gesicherte Daten vor. In einer Fall-KontrollStudie mit 2582 Kolorektalkarzinom-Patienten und 1798 gesunden Kontrollen, zwischen 60 und 79 Jahre alt, stellte sich nun Folgendes heraus: Innerhalb von fünf Jahren nach einer Polypektomie ist das Risiko, an einem Kolorektalkarzinom zu erkranken, signifikant reduziert. Dies traf auch für Patienten $\mathrm{zu}$, bei denen ein High-risk-Adenom entfernt wurde. Die Forscher schlagen demnach vor, die Nachuntersuchungen, auch nach der Entfernung von High-risk-Adenomen, im Fünf-Jahresabstand durchzuführen.

\section{Dank der Flavanole}

\section{Denk-fit mit Kakao}

Leichte kognitive Störungen sind offenbar mit einem simplen Rezept aufzuhalten oder sogar umzukehren - mit Kakao.

Die in der Kakaobohne enthaltenen Flavanole, Pflanzstoffe aus der „Familie“ der Flavonoide, sollen laut einer italienische Studie dem altersbedingten geistigen Abbau entgegenwirken.

Beweise dafür erhielten die Forscher in einem Praxistest mit 90 leicht kognitiv defizitären Senioren, die in drei Gruppen eingeteilt wurden. In Form eines Getränks erhielten die Probanden acht Wochen lang täglich jeweils 990 bzw. $45 \mathrm{mg} \mathrm{Ka-}$ kaoflavanole. Zu Beginn und acht Wochen später beurteilen die Geriater die geistigen Fähigkeiten ihrer Probanden: Die Senioren, die eine hohe oder mittlere Flavanoldosis zu sich genommen hatten, schnitten dabei insgesamt signifikant besser ab als zu Beginn der Studie, nicht so die Senioren mit niedriger Flavanoldosis. 